\title{
Unilateral Primary Lung Hypoplasia Diagnosed in Adulthood
}

\author{
Stamatis Katsenos MD PhD, Elvira-Markela Antonogiannaki MD, and Konstantinos Tsintiris MD
}

\begin{abstract}
Unilateral primary pulmonary hypoplasia is rare in adulthood. It is usually present in the neonatal period or in early childhood, and is characterized by a decreased number of bronchial segments and decreased/absent alveolar air space. Most patients have recurrent episodes of wheezing or pneumonia and severe respiratory distress leading to chronic respiratory failure, whereas some patients may occasionally be asymptomatic. Herein, we present a case of left lung hypoplasia in an asymptomatic 28-year-old male, who was admitted to the hospital for further investigation of an abnormal result on a chest radiograph that was obtained as part of a health evaluation for military service. A thorough workup, including imaging modalities and bronchoscopy, disclosed a hypoplastic left lung, which had gone undiagnosed for 25 years. Embryological, clinical, and diagnostic aspects are briefly discussed. Key words: primary lung hypoplasia;computed tomography;bronchoscopy. [Respir Care 2014;59(4):e47-e50. @ 2014 Daedalus Enterprises]
\end{abstract}

\section{Introduction}

Unilateral primary pulmonary hypoplasia is rare in adulthood. It is usually present in the neonatal period or in early childhood and is characterized by a decreased number of bronchial segments and decreased/absent alveolar air space. The majority of patients present with severe respiratory distress or repeated pulmonary infections and wheezing, whereas some may be completely asymptomatic. In this article, we present a case of left lung hypoplasia in an asymptomatic 28 -year-old male, who was admitted to the hospital for further investigation of an abnormal result on a chest radiograph obtained as part of a health evaluation for military service. The disorder had not been detected for 28 years.

\section{Case Report}

A 28-year-old male non-smoker was referred to our department for further evaluation of abnormal chest radio-

\footnotetext{
The authors are affiliated with the Department of Pneumonology, Army General Hospital of Athens, Athens, Greece.
}

Correspondence: Stamatis Katsenos MD PhD, Department of Pneumonology, Army General Hospital of Athens, 158 Mesogion \& Katehaki Avenues, 11525 Athens, Greece. E-mail: skatsenos@yahoo.gr.

DOI: $10.4187 /$ respcare. 02740 graph results. A plain chest radiograph is compulsory test for Greek military recruits as part of the health evaluation for their military service. The patient's medical history was unremarkable. He reported occasional mild episodes of the common cold without seeking any medical attention. A clinical examination of the chest revealed diminished movement of the thorax on the left side as well as the absence of breath sounds over the upper and middle left lung fields. Heart sounds were also heard on the left side. A posteroanterior chest radiograph showed opacification of the left hemithorax with decrease in its size and mediastinal displacement to the left with an increase in volume of right lung. The cardiac contours were indistinct (Fig. 1). Further imaging evaluation with multislice chest computed tomography (CT) scanning demonstrated a hypoplastic left lung with right lung hyperinflation and herniation to the contralateral side, as well as displacement of the mediastinum to the left side. Moreover, absence of the left main pulmonary artery and its branches into the left upper lobe and lingula as well as a decrease in size of the left main bronchus were noted. However, the left lower lobe artery with the corresponding lobe were present (Fig. 2). Fiberoptic bronchoscopy was then performed showing stenosis of the left main bronchus with absence of the left upper lobe and lingula bronchi as well as the left superior lower lobe segment (Fig. 3). The basal segments of the left lower lobe were rudimentary (Fig. 4). Furthermore, the right tracheobronchial tree was completely developed, but the orifices of the upper lobe and middle lobe bronchus were 


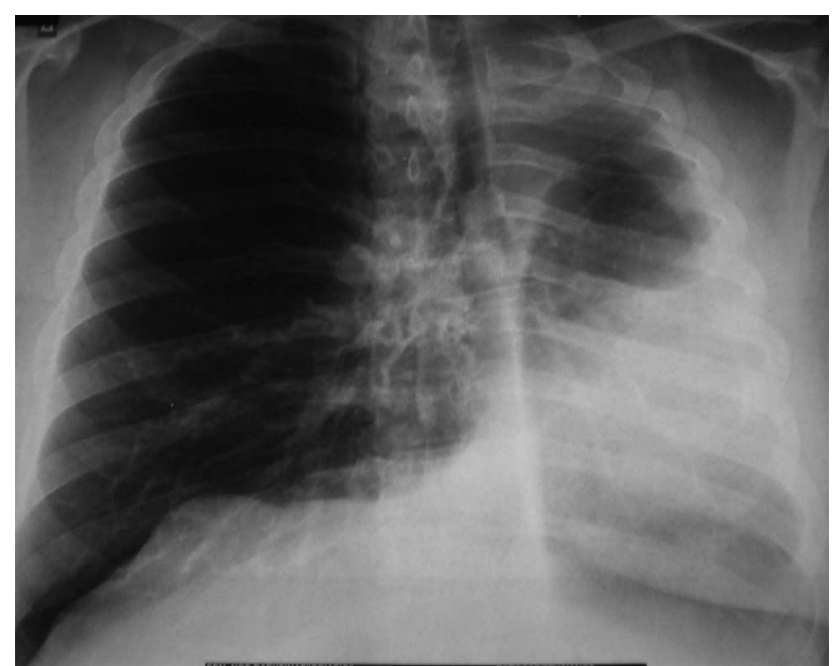

Fig. 1. Posteroanterior chest radiograph showing opacification of the left hemithorax with decrease in size and mediastinal shift to the left with an increase in the volume of the right lung. The heart outline is indistinct.

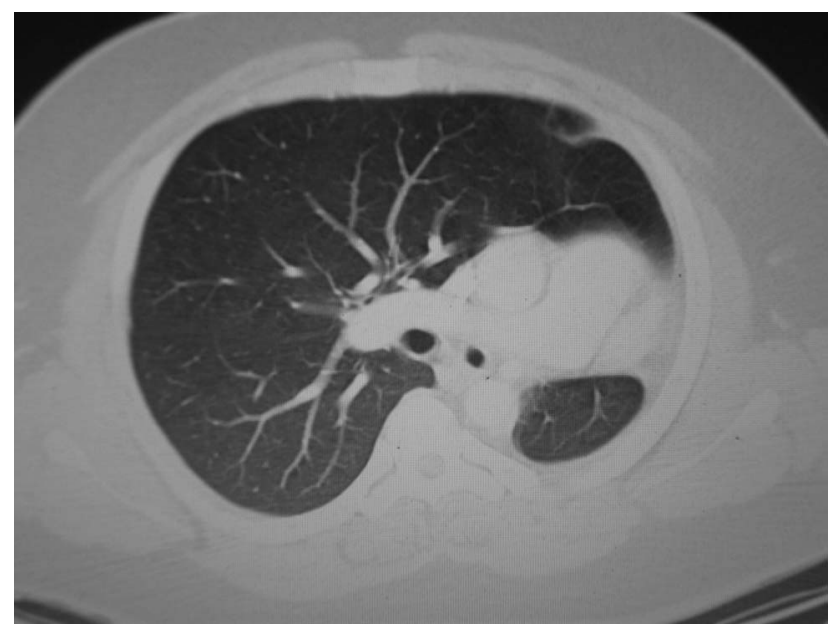

Fig. 2. CT showing hypoplastic left lung with right lung hyperinflation and herniation to the contralateral side, as well as displacement of the mediastinum to the left side. Absence of left main pulmonary artery as well as decreased size of left main stem bronchus.

in different positions than usual because of compensatory right lung expansion and consequent distortion of the bronchial structures. A ventilation-perfusion scan of the chest showed complete absence of ventilation and perfusion in the left upper lobe and lingula (Fig. 5). Pulmonary function tests demonstrated moderate-to-severe obstruction that was mildly responsive to inhaled bronchodilators. In particular, decreased values were noted in expiratory volumes (FVC $76 \%$ predicted, $\mathrm{FEV}_{1} 44 \%$ predicted, $\mathrm{FEV}_{1} / \mathrm{FVC}$ ratio $59 \%$ predicted, peak expiratory flow, $25 \%$ predicted). The measurement of static lung volumes revealed increased residual volume (200\% predicted) and total lung capacity

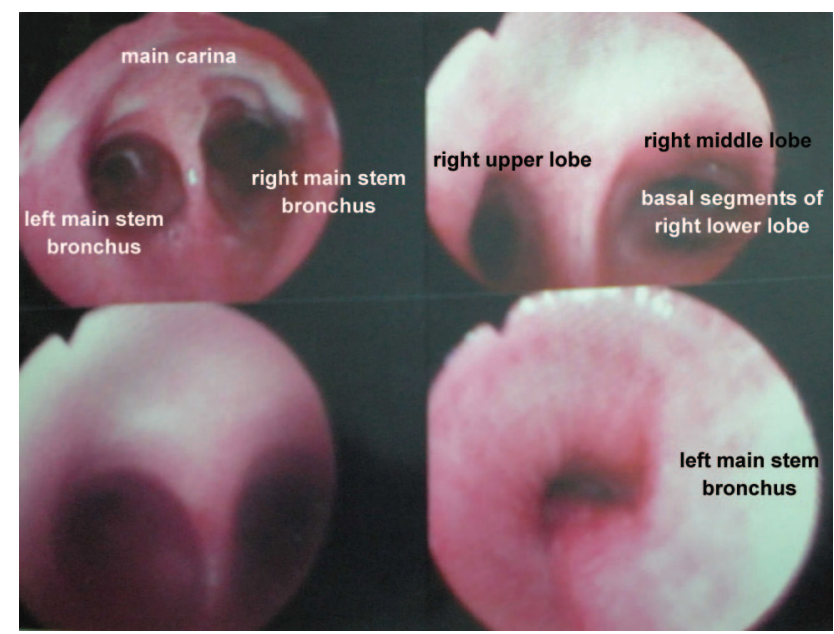

Fig. 3. Fiberoptic bronchoscopy showing stenosis of the left main stem bronchus, with absence of left upper lobe and lingula bronchi, as well as left superior lower lobe segment.

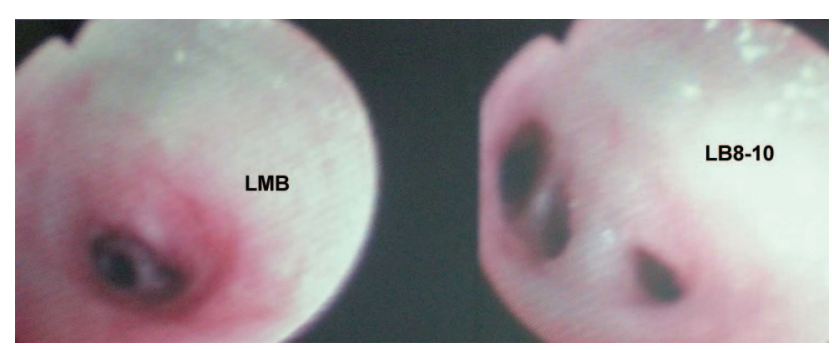

Fig. 4. Left: fiberoptic bronchoscopy showing left main stem bronchus. Right: fiberoptic bronchoscopy showing basal segments of the lower lobe.

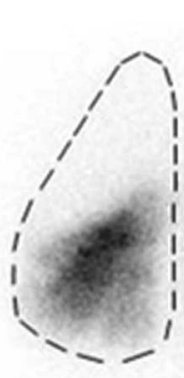

left lung

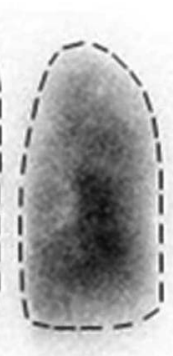

right lung

$$
\mid
$$

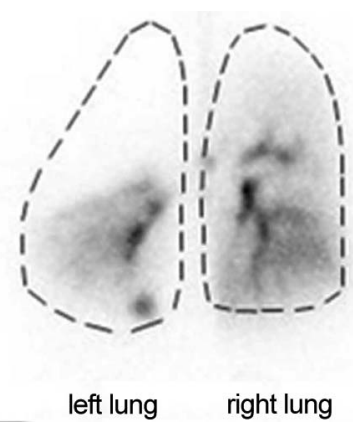

Fig. 5. Posterior scintigram showing perfusion only in left lower lobe (left) and ventilation only in left lower lobe (right).

(127\% predicted). Diffusing capacity of the lung was found to be moderately reduced ( $51 \%$ predicted). An additional workup including echocardiography and abdominal CT scan disclosed no coexistent congenital abnormalities. Therefore, a diagnosis of primary left lung hypoplasia was made, and it was recommended that the patient ask for medical help in the event of respiratory infections. In addition, inhaled $\beta_{2}$ agonists and steroids were administered 
to the patient despite their minor bronchodilating effect. Preventive vaccination against influenza virus and Pneumococcus was also recommended.

\section{Discussion}

Pulmonary underdevelopment has been classified into three groups by Lee et $\mathrm{al}^{1}$, Berrocal et $\mathrm{al}^{2}$, and Schneider and Schwalbe. ${ }^{3}$ In group 1 bronchus and lung are absent (agenesis), in group 2 a rudimentary bronchus is present and limited to a blind-end pouch without lung tissue (aplasia), and in group 3 there is bronchial hypoplasia with variable reduction of lung tissue (hypoplasia).

More specifically, pulmonary hypoplasia is defined as deficient or incomplete development of the lungs. The abnormality is characterized by the presence of both bronchi and alveoli in an underdeveloped lobe and may occur between the 4th and 24th gestational week. It can be categorized into two forms: primary and secondary. Most cases are usually secondary to conditions that limit fetal lung growth, whereas primary pulmonary hypoplasia is rare and is thought to be caused by an embryologic defect of the lung or vascular tissues or an in utero vascular accident. ${ }^{4}$ The true prevalence is unknown, but in cases of premature rupture of membranes at 15-28 weeks gestation the reported prevalence of pulmonary hypoplasia ranges from $9 \%$ to $28 \%$.

Primary unilateral pulmonary hypoplasia is usually encountered in a child presenting with life-threatening symptomatology (eg, early respiratory distress after birth, cyanosis, tachypnea, hypoxia, hypercapnia, and acidosis). However, it may be infrequently present in adults not producing any symptomatology thus rendering its diagnosis problematic. ${ }^{5}$ Nevertheless, some adult patients may present with repeated pulmonary infections and wheezing. ${ }^{6,7}$

Plain radiographs demonstrate decreased aeration of the affected hemithorax and a small thoracic cage. A common finding is a shift of the mediastinum to the side of the hypoplasia, accentuated during inspiration due to increased compensatory ventilation of the other lung. A retrosternal soft tissue density can also be visible on lateral chest films representing heart and mediastinum that are displaced into the anterior chest. A multidetector CT scan provides a noninvasive modality of confirming the diagnosis by clearly delineating the deformed and stunted pulmonary vasculature and bronchial tree. ${ }^{8,9}$ Bronchoscopy, ventilation-perfusion scanning, or CT-angiography can equally identify the absence of bronchovascular structures.

Differential diagnosis primarily includes congenital cystic adenomatoid malformation, pulmonary sequestration, congenital lobar emphysema, acquired lung collapse, and secondary pneumopathy with associated non-congenital bronchiectasis (pseudo-hypoplasia). ${ }^{2,10}$ However, there was no clinical or imaging evidence to support any of these entities in the present case. More frequently, pulmonary hypoplasia is secondary to other fetal developmental abnormalities, such as congenital diaphragmatic hernia, neuromuscular disorders, congenital heart diseases, thoracic cage anomalies (eg, asphyxiating thoracic dystrophy, Jeune syndrome), genitourinary tract anomalies (eg, Potter syndrome, oligohydramnios tetrad), chromosomal aberrations, and congenital pulmonary venolobar syndrome (Scimitar syndrome). These disorders occur in neonates and influence adversely morbidity and survival. ${ }^{11}$ However, it seems that patients with asymptomatic unilateral primary hypoplasia in adulthood exhibit long survival times due to compensatory hypertrophy of the contralateral lung that occupies the ipsilateral hemithorax. The most favorable survival has been observed in cases of left lung hypoplasia because of the satisfactory compensatory hypertrophy of the larger right lung. ${ }^{2,8}$ With regard to the present case, we speculate that lung hypoplasia should have occurred in the first 7-10 weeks of gestational age despite the fact that the individual had no symptoms referable to the chest for 28 consecutive years. It is quite likely that right lung compensatory expansion prevented the presence of chest symptomatology.

Pneumothorax and pulmonary hypertension are common serious complications. Pneumothorax often develops spontaneously or secondary to mechanical ventilation in infants who present with severe respiratory insufficiency in the first few hours of life. Last but not least pulmonary infections can cause life-threatening events in patients with this congenital malformation. The underdevelopment of the alveolar tissue results in a small fibrotic and nonfunctioning lung with associated surfactant deficiency and impaired mucociliary clearance, thus rendering the host susceptible to greater bacterial or viral proliferation.

In conclusion, unilateral primary lung hypoplasia is very rare in adulthood. The disease is usually established in the perinatal or neonatal period. Symptomatology is generally present despite the occasional accidental discovery of hypoplastic lung in an asymptomatic adult. Multidetector CT scanning is an instrumental imaging modality in establishing the diagnosis of pulmonary hypoplasia because it can discriminate the abnormalities of the bronchovascular structures.

\section{REFERENCES}

1. Lee EY, Dorkin H, Vargas SO. Congenital pulmonary malformation in pediatric patients: review and update on etiology, classification and imaging findings. Radiol Clin North Am 2011;49(5):921-948.

2. Berrocal T, Madrid C, Novo S, Gutiérrez J, Arjouilla A, GómezLeón N. Congenital anomalies of the tracheobronchial tree, lung and mediastinum: embryology, radiology, and pathology. Radiographics 2004;24(1):e17.

3. Schneider P, Schwalbe E. Die morphologie der missbildungen des menschen und der tiere. Jena, Germany: Fischer; 1912;3:812-822. 


\section{Unilateral Primary Lung Hypoplasia Diagnosed in Adulthood}

4. Porter H. Pulmonary hypoplasia. Arch Dis Child Fetal Neonatal Ed 1999;81(1):81F-83F.

5. Pathania M, Lali BS, Rathaur VK. Unilateral pulmonary hypoplasia: a rare clinical presentation. BMJ Case Rep 2013;2013.

6. Thomas RJ, Lathif HC, Sen S, Zachariah N, Chako J. Varied presentations of unilateral lung hypoplasia and agenesis: a report of four cases. Pediatr Surg Int 1998;14(1-2):94-95.

7. Comet R, Mirapeix RM, Marin A, Castaňer E, Sans J, Domingo C. Pulmonary hypoplasia in adults: embryology, clinical presentation and diagnostic methods. Our experience and review of the literature. Arch Bronconeumol 1998;34(1):48-51.
8. Georgescu A, Nuta C, Boudari S. 3D imaging in unilateral primary pulmonary hypoplasia in an adult: a case report. Case Rep Radiol 2011;2011:659586.

9. Beigelman C, Howarth NR, Chartrand-Lefebvre C, Grenier P. Congenital anomalies of the tracheobronchial branching patterns: spiral CT aspects in adults. Eur Radiol 1998;8(1):79-85.

10. Çay A, Sarihan H. Congenital malformation of the lung. J Cardiovasc Surg 2000;41(3):507-510.

11. Currarino G, Williams B. Causes of congenital unilateral pulmonary hypoplasia: a study of 33 cases. Pediatr Radiol 1985;15(1): $15-24$ 